DOI: https://doi.org/10.46296/yc.v5i9ucedespsoct.0125

\title{
ELABORACIÓN DE UN QUESO SEMIDURO DE LECHE DE CABRA A ESCALA DE LABORATORIO
}

\section{ELABORATION OF A SEMI HARD CHEESE FROM GOAT'S MILK ON A LABORATORY-SCALE}

\author{
García-Vergara Zenia ${ }^{1}$; Moreira-Vera David Wilfrido ${ }^{2}$; \\ Varela-De Moya Humberto Silvio ${ }^{3}$; Bidot-Fernández Adela ${ }^{4}$ \\ ${ }^{1}$ Profesor Instructor del departamento Ciencia y Tecnología de los Alimentos de la \\ Universidad de Camagüey "Ignacio Agramonte Loynaz". Camagüey, Cuba. Correo: \\ zenia.garcia@reduc.edu.cu. ORCID ID: https://orcid.org/0000-0001-6730-6758 \\ ${ }^{2}$ Docente Investigador Titular Escuela Superior Politécnica Agropecuaria de Manabí \\ ESPAM MFL. Calceta, Ecuador. ORCID ID: https://orcid.org/0000-0002-7020-4178 \\ ${ }^{3}$ Profesor Auxiliar del departamento Química de la Universidad de Camagüey "Ignacio \\ Agramonte Loynaz". Camagüey, Cuba. Correo: humberto.valera@reduc.edu.cu. \\ ORCID ID: https://orcid.org/0000-0002-6632-3182 \\ ${ }^{4}$ Centro de investigaciones para el mejoramiento animal de la Ganadería Tropical \\ (CIMA-GT). La Habana, Cuba. Correo: abidot@infomed.sld.cu.
}

\begin{abstract}
Resumen
La leche de cabra es un alimento natural con unas características y propiedades que la hacen beneficiosa para la salud. El objetivo de la investigación fue elaborar un queso de cabra semiduro con las especificaciones de calidad en cuanto a humedad y grasa en extracto seco. Se realizaron tres producciones de queso de cabra a escala de laboratorio con tres réplicas de cada una de las determinaciones fisicoquímicas para evaluar la calidad de la leche, el suero y al producto terminado. La evaluación sensorial se realizó comparando las descripciones realizadas en las especificaciones de calidad de la norma. Los resultados obtenidos en los análisis de la leche mostraron comportamientos regulares, para la acidez y la materia grasa el coeficiente de variación arrojó valores por debajo del $10 \%$, y la densidad en las diferentes producciones cercanas a la media aritmética. En el caso del suero la acidez cumplió con lo especificado según lo normado y los resultados del coeficiente de variación en la materia grasa indican que los datos obtenidos tienen un comportamiento regular. Asimismo, la materia grasa del queso indica que en las tres producciones siguen un comportamiento regular, así como el análisis de la humedad. El por ciento de grasa en extracto seco mostró que para la segunda y tercera producción se estuvieron valores conforme a la norma para los quesos semiduros tipo Coral. En cuanto a la evaluación sensorial del queso de cabra semiduro obtenido en las tres producciones se arrojaron criterios conformes a lo normado.
\end{abstract}

Palabras claves: queso; queso semiduro; leche de cabra; producción de queso.

\begin{abstract}
Goat milk is a natural food with characteristics and properties that make it beneficial for health. The objective of the research was to produce a semi-hard goat cheese with quality specifications in terms of moisture and fat in dry matter. Three laboratory-scale goat cheese productions were carried out with three replications of each of the physicochemical determinations to evaluate the quality of the milk, the whey and the finished product. The sensory evaluation was carried out by comparing the descriptions made in the quality specifications of the standard. The results obtained in the milk analyzes showed regular behaviors, for acidity and fat, the coefficient of
\end{abstract}

Información del manuscrito:

Fecha de recepción: 26 de julio de 2021.

Fecha de aceptación: 29 de septiembre de 2021.

Fecha de publicación: 11 de octubre de 2021. 
variation showed values below $10 \%$, and the density in the different productions close to the arithmetic mean. In the case of whey, the acidity complied with what was specified according to the norm and the results of the coefficient of variation in fat indicate that the data obtained had a regular behavior. Likewise, the fat of the cheese indicates that the three productions follow a regular behavior, as well as the moisture analysis. The percent of fat in dry extract showed that for the second and third production values were in accordance with the norm for Coral type semihard cheeses. Regarding the sensory evaluation of the semi-hard goat cheese obtained in the three productions, criteria conforming to the norm were thrown.

Keywords: cheese; semi-hard cheese; goat milk; cheese production.

\section{Introducción}

La leche de las distintas especies de rumiantes, bien directo o como productos derivados, constituye un alimento de singular importancia para el ser humano durante toda su vida. Este alimento puede considerarse fuente de macro y micronutrientes y un cierto número de compuestos activos, que juegan un papel importante, nutritivo y de protección.

En este sentido la leche de cabra, así como los productos derivados de esta, se consideran de acuerdo a su composición específica, de un gran interés dentro de la nueva tendencia por consumir alimentos de una alta calidad nutritiva, es por ello que en países desarrollados se están enfocando en la producción de nutracéuticos a partir de la leche de cabra. (Núñez, 2018).
La leche de cabra, Europa representa el $16,6 \%$ de la producción mundial, siendo España el segundo productor de Europa 14,1 \%; 328 litros/animal y, junto a Francia y Grecia, agrupa el $70 \%$ del censo caprino lechero y más del $80 \%$ de la producción de leche de cabra europea (Pulina et al., 2018; Ureña et al., 2021). En cuanto a la producción de leche de cabra, el principal país es India (27 \%) seguido por Bangladesh (14\%) y Sudán (8\%) (Schettino et al., 2018). La producción de leche de cabra se ha incrementado a nivel mundial debido a los beneficios que aporta a la salud, por su alto contenido de ácidos grasos C6:0 y C10:0, la carencia de aglutinina y el menor tamaño del glóbulo de la grasa, lo que se ha relacionado con una mayor digestibilidad. También entre los ácidos grasos de la grasa láctea se encuentra el ácido linoleico 
conjugado, que ha despertado un gran interés por sus efectos anticancerígenos, prevención de la arterosclerosis y la respuesta inmune en la salud humana (Bidot, 2017; Llerena et al., 2017; Núñez, 2018).

La leche de cabra posee unas características altamente beneficiosas que le confieren un alto interés como alimento y objeto de investigación.

Numerosas investigaciones han demostrado la condición de alimento funcional de esta leche; más allá de su valor nutritivo básico, la leche de cabra posee compuestos biológicamente activos en su forma natural que son beneficiosos para la salud en prevención, mantenimiento o tratamientos terapéuticos en diversas enfermedades (Tripathi, 2015).

Dentro de los compuestos bioactivos de la leche de cabra se encuentran las poliaminas, nucleótidos, aminoácidos libres, ácidos grasos de cadena mediana, ácidos grasos poliinsaturados. Los beneficios terapéuticos potenciales del consumo de la leche de cabra y de sus productos lácteos, como anticarcinogénicos, antivirales, y en la prevención de enfermedades cardiovasculares, han resultado en un aumento de sus ingestas (Tripathi, 2015).

Debido a sus propiedades, su composición y a la alta aceptación que ha alcanzado la leche de cabra en el mercado mundial se pueden encontrar una gran variedad de productos derivados de esta. Los derivados más frecuentes son leche de cabra entera pasteurizada, leche en polvo, leche esterilizada, batidos de leche con sabores, yogurt natural, cuajada, dulces de leche y quesos de varios tipos. El queso es una forma de conservación de la leche. Es una fuente de nutrientes ya que concentra la proteína, calcio y demás nutrientes y su tiempo de vida es mayor que el de la leche fresca (Sandoval, 2018).

Se entiende por queso el producto fresco o maduro, sólido o semisólido, obtenido por separación del suero después de la coagulación de la leche natural, de la desnatada total o parcialmente, de la nata, del suero de mantequilla o de una mezcla de algunos o de todos estos productos por la acción del cuajo u otros coagulantes apropiados, con o sin 
hidrólisis previa de la lactosa (Sandoval, 2018).

En consecuencia, el queso no es más que leche muy concentrada, por eso las características de la leche utilizada definirán, en gran parte, las del queso resultante. El cuajo contiene quimosina, una enzima que permite que la capa-caseína responsable de mantener las micelas de caseína en la leche separada se inactive. En el caso de los microorganismos las bacterias y los hongos son los que darán las peculiaridades a cada queso al modificar las proteínas y grasas presentes en la leche, dando lugar a nuevos compuestos con sabores y aromas característicos. De ahí que, el queso se considera un alimento de fácil fabricación, elaborado a partir de la leche cuajada de vaca, oveja, búfala, camella y cabra (Sandoval, 2018).

Mundialmente se producen diferentes variedades de quesos de cabra, entre éstos puede citarse el queso Crottin de Chavignol, producido en Francia empleando cuajada cruda de leche entera de cabra; éste se caracteriza por ser un queso madurado de pasta blanda. Los quesos pueden elaborarse con leche entera, parcialmente descremada, semidescremada, descremada, crema o doble crema y entre sus clasificaciones se conocen los frescos, procesados y curados. Este último son los quesos de pasta más dura sometidos a un proceso de maduración mediante la adición de microorganismos bajo condiciones controladas de tiempo, temperatura y humedad. Estos microorganismos provocan en ellos cambios bioquímicos y físicos, que les confieren la consistencia y el sabor característicos (Sandoval, 2018).

Los quesos semiduros de cabra se consideran superiores a los otros tipos de queso ya que tiene una mayor vida útil debido a su menor contenido de humedad. Las producciones de estos quesos en Cuba son insuficientes para cubrir las necesidades de la industria turística y de la población. Sin embargo, se conocen territorios en los que se producen valores significativos de esta leche, por ejemplo, en el municipio Jimaguayú de la provincia de Camagüey.

En el caso de la provincia de Camagüey, que es un territorio ganadero, se realizan varios proyectos referidos al incremento de 
las producciones derivadas de la leche de cabra, así como de su calidad para el consumo. Un ejemplo de estos lo constituye la que es desarrollada por la Universidad de Camagüey en conjunto con el centro de Investigación para el mejoramiento animal de la ganadería tropical. Esta investigación responde al proyecto Aplicación de tecnologías que contribuyan al incremento productivo de la leche caprina y al desarrollo de producciones secundarias en la cadena agroindustrial.

La presente investigación se corresponde con una de las tareas del mencionado proyecto que tiene como antecedentes la elaboración de queso fresco de leche de cabra en la Universidad de Camagüey mediante un diseño de un procedimiento tecnológico. Sin embargo, aún no se ha experimentado la obtención de queso semiduro a partir de leche caprina bajo las condiciones del proyecto, por lo que se define como problema de investigación: ¿Cómo obtener un queso de cabra semiduro que cumpla con las especificaciones de calidad requeridas? A partir de los presupuestos expuestos el objetivo de la investigación es elaborar un queso de cabra semiduro que cumpla con las especificaciones de calidad en cuanto a humedad $y$ grasa en extracto seco.

\section{Metodología}

La investigación se llevó a cabo en las fábricas Combinado Lácteo de Camagüey y La Vaquita. En ella se realizaron tres producciones de queso de cabra a nivel de laboratorio siguiendo de forma general, los pasos tecnológicos expuestos en la norma de empresa Quesos semiduros, Coral y Guamá. Proceso tecnológico (NEIAL1609.70 (1999).

Entre las técnicas usadas se encuentran la del análisis documental y la observación. Los métodos que se emplearon en la realización de este informe fueron: métodos teóricos (análisis y síntesis) y los empíricos (observación científica). Se utilizaron además los métodos de medición para obtener la información numérica necesaria y los métodos experimentales.

Con el fin de evaluar la calidad de la leche para la elaboración de queso, se realizaron tres réplicas de cada 
una de las determinaciones físicoquímicas siguientes: densidad (NC 119 (2006), acidez (NC 71 (2000) y contenido de materia grasa (NC ISO2446 (2003).

Las determinaciones físico-químicas realizadas al suero fueron: materia grasa (NC ISO-2446 (2003) y acidez (NC 71 (2000).

Las determinaciones físico-químicas al producto terminado fueron: humedad (NC 78-17 (1989) y contenido de materia grasa (NC 7818(1989).

Cálculo del porciento de grasa en extracto seco (GES) aplicando la siguiente expresión:

$\%$ GES $=\frac{\% \text { grasa en el queso }}{100-\% \text { de humedad en el queso }} \cdot 100$

Para ello se utilizó la media aritmética del porciento de grasa y de humedad obtenido del análisis estadístico realizado a las réplicas de cada determinación fisicoquímica al producto terminado.

La evaluación sensorial se realizó comparando las descripciones realizadas en las especificaciones de calidad de la norma de empresa (NEIAL 1609.64 (1997) Quesos semiduros, mediante un análisis descriptivo donde se seleccionaron los atributos adecuados para la aplicación (forma, corteza, masa y ojos).

Los resultados de las determinaciones fisicoquímicas de la leche y del queso de cabra fueron organizados utilizando el programa Microsoft Excel 2019. Para comprobar los parámetros evaluados se efectuó un análisis estadístico. Para ello se escogieron como variables los porcientos de humedad y GES del queso, determinando media, desviación estándar y coeficiente de variación, con la utilización del paquete estadístico Statgraphics Centurion versión $\mathrm{XV}$.

Procedimiento de elaboración de queso semiduro de cabra a escala de laboratorio

Se realizó un estudio previo de los procesos de elaboración de queso semiduro a partir de leche de vaca descritos en la norma de empresa NEIAL1609.70 (1999). Este fue adaptado a las características de la materia prima fundamental (leche de cabra) y a las condiciones de trabajo del laboratorio donde se realizaron los experimentos. 
Recepción de la materia prima: Se utilizó en todas las elaboraciones leche fresca e íntegra de cabra con menos de tres horas de ordeño de un mismo día, procedente de un productor independiente del municipio de Jimaguayú. Este ordeño se efectuaba a las 6:00 am y luego la leche se transportaba hacia el Combinado Lácteo de Camagüey a temperatura de 15 a $20{ }^{\circ} \mathrm{C}$. En la primera producción se utilizaron 8,75 $\mathrm{L}$ de la leche mientras que en la segunda y la tercera $5 \mathrm{~L}$. Luego se comprobó la calidad de esta través de los análisis físico-químicos.

Filtración: En esta fase la leche se filtró con un paño de gasa esterilizada para eliminar cualquier tipo de impurezas que hayan podido pasar a la leche durante el ordeño y prevenir que se contamine el producto final.

Pasteurización: Se elevó la temperatura de la leche hasta $65^{\circ} \mathrm{C}$ por 30 minutos (pasteurización lenta) en baño de María.

Enfriamiento: La leche después de pasteurizada fue enfriada colocando el recipiente en la que se encontraba, en agua helada hasta lograrse la temperatura de $32^{\circ} \mathrm{C}$.
Adición de cultivo: Se añadió el cultivo con el objetivo de incrementar la acidez de la leche. La cantidad a añadir fue de $1 \%$ de la cantidad de leche utilizada. En la primera producción se empleó $87,5 \mathrm{~mL}$ mientras que en la segunda y la tercera producción $50 \mathrm{~mL}$. Luego se dejó en reposo durante 30 minutos.

Adición de cuajo: Para facilitar la coagulación y obtener mayor rendimiento en el queso se añadió 1 $\mathrm{mL}$ de cuajo/10 $\mathrm{L}$ de leche. La cantidad de cuajo a utilizar fue de una parte del cuajo por diez partes de agua. En la primera producción se empleó $0,87 \mathrm{~mL}$ mientras que en la segunda y la tercera producción $1 \mathrm{~mL}$.

Corte de la cuajada: La misma se cortó de forma horizontal y vertical utilizando paletas de acero inoxidable para la elaboración de queso. Inmediatamente se procede al desuerado.

Desuerado: Los granos de la cuajada, una vez separados del suero, se sustrajeron cuidadosamente, evitando eliminar pequeñas porciones de queso que influyeran negativamente en el rendimiento. 
Salado: Se preparó $1 \mathrm{~L}$ de salmuera de densidad $1,160 \mathrm{~g} / \mathrm{cm}^{3}$, la misma se pasteurizó y se filtró antes de ser añadida a la cuajada a razón de 100 $\mathrm{mL} / 10 \mathrm{~L}$ de leche.

Pre-prensado: Esta operación tiene como objetivo aprisionar la cuajada con el fin de eliminarle la mayor cantidad de suero posible. Primeramente, se utilizó una masa de 0,27 Kg por kilogramo de queso. Transcurrido 5 minutos, se duplicó esta masa por otros 5 minutos.

Prensado: Tiene como objeto compactar la masa del queso y eliminar el suero sobrante. Se procedió de la siguiente forma: primera producción $8 \mathrm{Kg}$ de masa por 60 minuto, la segunda producción $10 \mathrm{Kg}$ de masa por 60 minuto y en la tercera producción se varió la masa y el tiempo de la siguiente manera, se utilizó inicialmente una masa de $5 \mathrm{Kg}$ por
60 minutos, después $10 \mathrm{Kg}$ por 30 minutos y luego $20 \mathrm{Kg}$ por 30 minutos.

Maduración: El tiempo de maduración de los quesos para la primera producción fue de 30 días NEIAL1609.70 (1999) en la segunda 25 días y en la tercera 20 días (Fernández 2014).

\section{Resultados}

Análisis de la acidez en leche: Los resultados obtenidos en el procesamiento estadístico para los valores de acidez se muestran en la tabla 1. Se evidencia que los datos se encuentran cercanos a la media ya que la desviación estándar para las tres producciones es de 0,01 . Por otro lado, el comportamiento de estos es regular ya que el coeficiente de variación arrojó valores por debajo del $10 \%$.

Tabla 1. Análisis estadístico de las determinaciones de acidez materia grasa y densidad de la leche.

\begin{tabular}{|c|c|c|c|c|c|c|c|}
\hline \multirow{2}{*}{\multicolumn{2}{|c|}{$\begin{array}{l}\text { Determinaciones } \\
\text { Producciones }\end{array}$}} & \multicolumn{3}{|c|}{ Acidez } & \multicolumn{3}{|c|}{ Materia grasa } \\
\hline & & 1 & 2 & 3 & 1 & 2 & 3 \\
\hline \multirow{3}{*}{ Leche } & Media & 0,123 & 0,103 & 0,11 & 2,783 & 1,283 & 0,7 \\
\hline & $S$ & 0,015 & 0,015 & 0,02 & 0,028 & 0,028 & 0,01 \\
\hline & $\mathrm{CV}$ & 12,385 & 14,782 & 18,181 & 1,037 & 2,249 & 1,428 \\
\hline \multicolumn{2}{|c|}{$\begin{array}{l}\text { Referencia NEIAL } \\
1609.70(1999)\end{array}$} & \multicolumn{3}{|c|}{$0,13-0,17 \%$} & \multicolumn{3}{|c|}{$0,20 \%-0,30 \%$} \\
\hline
\end{tabular}




\begin{tabular}{|c|c|c|c|c|}
\hline \multirow{2}{*}{\multicolumn{2}{|c|}{$\begin{array}{l}\text { Determinaciones } \\
\text { Producciones }\end{array}$}} & \multicolumn{3}{|c|}{ Densidad } \\
\hline & & 1 & 2 & 3 \\
\hline \multirow{3}{*}{ Leche } & Media & 1,0302 & 1,030 & 1,0315 \\
\hline & $S$ & 0,0002 & 0,001 & 0,0005 \\
\hline & $\mathrm{CV}$ & 0,024 & 0,097 & 0,048 \\
\hline \multicolumn{2}{|c|}{$\begin{array}{l}\text { Referencia NEIAL } \\
1609.70 \text { (1999) }\end{array}$} & \multicolumn{3}{|c|}{$1,029 \mathrm{~g} / \mathrm{L}-1,033 \mathrm{~g} / \mathrm{L}$} \\
\hline
\end{tabular}

Fuente: Autores (2021).

Análisis de materia grasa: Los resultados de las réplicas de cada una de las producciones para la materia grasa, se encuentran en concordancia con lo especificado en la norma para esta variable. La desviación estándar obtenida para cada producción muestra valores entre 0,028 y 0,275 , lo que indica que los resultados de las réplicas se encuentran cercanos a su valor promedio. En cuanto al coeficiente de variación, los valores se encontraban por debajo del $10 \%$, indicando esto que los datos obtenidos de materia grasa en cada una de las producciones, tienen un comportamiento regular.

Análisis de la densidad de la leche: De acuerdo a la tabla 1. que expresa el análisis estadístico realizado a las determinaciones de densidad, la media de los resultados para esta variable arrojó que los valores obtenidos en todas las producciones cumplen con lo especificado en la norma. En cuanto al comportamiento de los resultados de la desviación estándar y del coeficiente de variación (entre 0,0002-0,001 y 0,024\%-0,097\% respectivamente), se puede determinar que los datos de las producciones se encuentran cercanos a la media aritmética y tienen un comportamiento regular.

Análisis acidez del suero: Como se observa en la tabla 2., según la desviación estándar, los datos están próximos al valor de la media aritmética, sin embargo, estos no tienen un comportamiento de forma irregular ya que el resultado del coeficiente de variación supera el 10 $\%$ en las tres producciones. Aun así, la media de la acidez del suero según lo normado, cumple con lo especificado.

Análisis materia grasa del suero: Los resultados del análisis estadístico a las determinaciones fisicoquímicas 
realizadas al suero, se muestran en la tabla 2. La desviación estándar de la primera y segunda producción es de 0,028 mientras que el de la tercera es 0,01 , lo que significa que los datos de las tres producciones son cercanos al valor de la media aritmética. Por su parte, los resultados del coeficiente de variación (1,037\%-2,249\%) indican que los datos obtenidos de grasa tienen un comportamiento regular.

Tabla 2. Análisis estadístico de las determinaciones de acidez, materia grasa del suero y humedad del queso.

\begin{tabular}{|c|c|c|c|c|c|c|c|}
\hline \multirow{2}{*}{\multicolumn{2}{|c|}{\begin{tabular}{|l} 
Determinaciones \\
Producciones
\end{tabular}}} & \multicolumn{3}{|c|}{ Acidez } & \multicolumn{3}{|c|}{ Materia grasa } \\
\hline & & \multirow{2}{*}{$\begin{array}{l}1 \\
0,123\end{array}$} & \multirow{2}{*}{\begin{tabular}{|l|}
2 \\
0,103
\end{tabular}} & \multirow{2}{*}{\begin{tabular}{|l|}
3 \\
0,11
\end{tabular}} & \multirow{2}{*}{\begin{tabular}{|l}
1 \\
2,783
\end{tabular}} & \multirow{2}{*}{\begin{tabular}{|l}
2 \\
1,283
\end{tabular}} & \multirow{2}{*}{$\begin{array}{c}3 \\
0,7\end{array}$} \\
\hline \multirow{3}{*}{ Suero } & Media & & & & & & \\
\hline & $\mathrm{S}$ & 0,015 & 0,015 & 0,02 & 0,028 & 0,028 & 0,01 \\
\hline & $\mathrm{CV}$ & 12,385 & 14,782 & 18,181 & 1,037 & 2,249 & 1,428 \\
\hline \multicolumn{2}{|c|}{$\begin{array}{l}\text { Referencia NEIAL } \\
1609.70 \text { (1999) }\end{array}$} & \multicolumn{3}{|c|}{$0,10 \%-0,12 \%$} & \multicolumn{3}{|c|}{$0,20 \%-0,30 \%$} \\
\hline \multicolumn{2}{|c|}{ Determinaciones } & \multicolumn{3}{|c|}{ Humedad } & \multicolumn{3}{|c|}{ Materia grasa } \\
\hline \multicolumn{2}{|c|}{ Producciones } & 1 & 2 & 3 & 1 & 2 & 3 \\
\hline \multirow{3}{*}{ Queso } & Media & 47,3 & 36,2 & 33,67 & 0,14 & 0,15 & 0,14 \\
\hline & $S$ & 1,101 & 0,288 & 2,516 & 0,01 & 0,01 & 0,01 \\
\hline & $\mathrm{CV}$ & 2,330 & 0,798 & 7,475 & 7,14 & 6,67 & 7,14 \\
\hline \multicolumn{2}{|c|}{$\begin{array}{l}\text { Referencia NEIAL } \\
1609.70 \text { (1999) }\end{array}$} & \multicolumn{3}{|c|}{$\leq 46 \%$} & & & \\
\hline
\end{tabular}

Fuente: Autores (2021).

Análisis materia grasa del queso: Según los resultados de las determinaciones fisicoquímicas realizadas al queso, los coeficientes de variación obtenidos en el análisis estadístico de la tabla 2., indican que los datos de las tres producciones siguen un comportamiento regular. Además, la desviación estándar obtenida en la segunda producción indica que sus datos están próximos a la media aritmética mientras que los de la primera y tercera producción están más distantes respectivamente.

Análisis humedad del queso: Los resultados del análisis estadístico a las determinaciones humedad en el queso se muestran en la tabla 2. Se puede señalar que para la segunda producción se obtuvo un 36,2 \% y para la tercera un $33,67 \%$ de 
humedad respectivamente, lo que concuerda con la norma NEIAL1609.64 (1997) la cual expone las especificaciones de calidad del queso semiduro Coral de un $46 \%$ máximo de humedad.

Cálculo del \% de grasa en extracto seco: Los resultados del \% GES de las distintas producciones fue la siguiente: primera $18 \%$, segunda $41,5 \%$ y la tercera $49,2 \%$. La segunda y la tercera estuvieron conforme a la norma NEIAL1609.64 (1997) que plantea para los quesos semiduros tipo Coral un valor mínimo del $40 \%$, no así en la primera producción.

Evaluación sensorial: En cuanto a la corteza, se describieron las características de esta, en los quesos de la primera y segunda producción de igual forma que la norma. Por su parte, en la tercera producción el color era menos intenso que el de las producciones anteriores. Esto pudo haber estado ocasionado por el tiempo de maduración que comparado con las otras producciones fue menor.

En cuanto el atributo ojos, los quesos cumplen con lo establecido en la norma presentándose distribuidos irregularmente por toda la masa. La característica consistencia de la segunda y la tercera producción estuvo conforme a la norma, no así en la primera producción, donde este queso era firme y resistente al corte. Esto se debe a que este queso posee menor contenido de grasa que los demás, provocando que sea menos pastosa la masa y más resistente al corte. En el caso de las características color, olor y el sabor las tres producciones cumplieron con lo establecido en la norma.

\section{Conclusiones}

Se obtuvo un queso de cabra semiduro que cumple con las especificaciones físico-químicas en cuanto a humedad y grasa en extracto seco.

Los resultados obtenidos en los análisis de la leche de cabra muestran comportamientos regulares, para la acidez y la materia grasa el coeficiente de variación arrojó valores por debajo del $10 \%$, y la densidad en las diferentes producciones cercanas a la media aritmética. La leche de cabra empleada para la producción de los quesos analizados cumplió con los 
requisitos de calidad establecidos en las normas.

En el caso del suero la acidez cumple con lo especificado según lo normado y los resultados del coeficiente de variación en la materia grasa indican que los datos obtenidos tienen un comportamiento regular. Asimismo, la materia grasa del queso indica que en las tres producciones siguen un comportamiento regular, así como el análisis de la humedad. El por ciento de grasa en extracto seco muestra que para la segunda y tercera producción se estuvieron valores conforme a la norma para los quesos semiduros tipo Coral.

En cuanto la evaluación sensorial del queso de cabra semiduro obtenido en las tres producciones se arrojaron criterios conformes a lo normado en cuanto a las características color, olor y sabor.

\section{Bibliografía}

Bidot, A. (2017). Composición, cualidades y beneficios de la leche de cabra: revisión bibliográfica. Revista De Producción Animal, 29 (2), 3241. Recuperado de https://revistas.reduc.edu.cu/i ndex.php/rpa/article/view/168 6

Cuba, Ministerio de la Industria Alimentaria. (1999). NEIAL 1609.70. Quesos semiduros Coral y Guamá. Proceso tecnológico. La Habana, Cuba: MINAL.

Cuba, Ministerio de la Industria Alimentaria. (2000). NC 71. Leche. Determinación de acidez. La Habana, Cuba: MINAL.

Cuba, Ministerio de la Industria Alimentaria. (2006). NC 119. Leche. Determinación de densidad. La Habana, Cuba: MINAL.

Cuba, Ministerio de la Industria Alimentaria. (2003). NC ISO2446. Leche. Determinación de materia grasa. La Habana, Cuba: MINAL.

Cuba, Ministerio de la Industria Alimentaria. NC 78-17. Queso. Determinación de humedad. La Habana, Cuba: MINAL.

Cuba, Ministerio de la Industria Alimentaria. (1989). NC 7817. (1989). NC 78-18. Queso. Determinación de materia grasa. La Habana, Cuba: MINAL.

Cuba, Ministerio de la Industria Alimentaria. (1997). NC 1609.64. Especificaciones de 
calidad. Quesos semiduros. La Habana, Cuba: MINAL.

Llerena, C., Loor, C., Suescun, N. \& Molina, G. (2017). Desarrollo de una leche de cabra fermentada empleando I. acidophilus en co-cultivo con las Bal del yogur. Revista Alimentos Hoy, 25, (41), 7795. ISSN: 2027-291X. Recuperado de: https://alimentoshoy.acta.org. co/index.php/hoy/article/view/ 449

Núñez, M. T. (2018). Las bondades para la salud de la leche de cabra y su potencial para producir alimentos funcionales. Revista AGROLLANÍA, 16 (2), 37-40. ISSN: 1690-8066.

Recuperado de: http://www.postgradovipi.50w ebs.com/archivos/agrollania/2 018_esp/Articulo\%207.pdf

Pulina, G., Milán, M. J., Lavín, M. P., Theodoridis, A., Morin, E., Capote, J., Thomas, D. L., Francesconi, A. H. D. \& Caja, G. (2018). Invited review: Current production trends, farm structures, and economics of the dairy sheep and goat sectors. J. Dairy Sci, 101, 6715-6729. ISSN: 00220302. Recuperado de: https://doi.org/10.3168/jds.20 17-14015

Sandoval, J. H. (2018). Evaluación de los riesgos físico-químicos y microbiológicos en la producción de quesos saborizados en la Cooperativa de producción Agropecuaria Chone Ltda. Tesis de maestría no publicada, Escola Superior de Turismo e Tecnología do Mar, Instituto Politécnico de Leiria, Leiria, Portugal.

Tripathi, M. K. (2015). Comforts in quality and production of goat milk. Advances in Dairy Research, 3, 1-2. ISSN: 2329888X. Recuperado de: https://doi.org/10.4172/2329$888 \mathrm{x} .1000 \mathrm{e} 115$

Schettino, B. S., Gutiérrez, R., Vega, S., Escobar, A., Pérez, J. J. \& González, M. (2018). Composición láctea y perfil de ácidos grasos en leche de cabra de Guanajuato, México. Revista de Salud Animal, 40 (2), 1-14. E-ISSN: 2224-4700. Recuperado de http://scielo.sld.cu/scielo.php ?script=sci_abstract\&pid=S02 53-

$570 \times 2018000200001 \& \operatorname{lng}=\mathrm{es}$ \&nrm=iso

Ureña, L.P., Colombo, S., López, A.L. \& Ruiz, FA. (2021). Demanda de productos animales sostenibles: leche de cabra de pastoreo. Archivos de Zootecnia, 70 (269), 60-70. ISSN: 18854494. Recuperado de: https://www.uco.es/servicios/ ucopress/az/index.php/az/arti cle/view/5419/3423 